\title{
Définitude et quantification universelle
}

\author{
Francis Corblin \\ Université Paris-Sorbonne \\ \& Institut Jean Nicod (CNRS-ENS-EHESS)
}

Francis.Corblin@paris-sorbonne.fr

\section{Résumé :}

Dans la plupart des théories du défini la notion de maximalité ou d'exhaustivité joue un rôle essentiel. La première question qui se pose à ce propos est d'ordre explicatif. Il y a d'autres formes linguistiques de la quantification universelle et il y a d'autres points de vue pour caractériser la définitude, notamment l'anaphore et la deixis. La seconde question qui se pose est d'ordre empirique. A peine at-on posé ou concédé que la définitude était proche de la quantification universelle, que les contreexemples se présentent. On soutient dans cette communication que la théorie du défini est indépendante, en principe de la notion de quantification universelle et d'exhaustivité, et on s'attache à établir avec précision les raisons pour lesquelles il semble en être autrement. Ce travail discute notamment les approches antérieures de Dowty (1987) et Brisson (2003) et utilise la typologie des prédicats introduits dans Corblin (2008) pour étayer une proposition d'ensemble.

L'idée centrale est que le défini est un désignateur (un terme) utilisant son contenu descriptif pour identifier (Corblin 1987). Ce mode d'identification détermine un premier lien à l'universalité : le défini le $\mathrm{x}$ désigne nécessairement tous les $\mathrm{x}$ de son domaine d'interprétation ; ce sont les limites noncontraintes de ce domaine, pragmatiquement déterminé qui l'éloignent de l'universel strict.

En tant que désignateur, et au même titre que le nom propre, le défini entre, par ailleurs, dans la prédication, ce qui détermine d'autres associations à l'universalité.

Le défini réfère à un individu qui peut être vu comme un tout, et il existe un mode de prédication « individuelle » qui assigne le prédicat à l'individu dénoté. La relation à l'universalité est déterminée, prédicat par prédicat, selon les inférences qu'il autorise quant à la satisfaction du prédicat par les parties de l'individu.

Le défini réfère à un individu qui, d'autre part, peut être vu comme un ensemble de parties et fait l'objet de prédications quantifiées au moyen d'expressions lexicales (tous). Seuls les prédicats holistes qui s'appliquent à un individu considéré dans toutes ses parties n'admettent pas la prédication quantificationnelle. Le défini, comme les conditionnelles admet des interprétations quantificationnelles sans quantificateur lexical réalisé. Comme pour les conditionnelles, le quantificateur restitué s'interprète comme « universel ou quasi-universel ».

On s'attache à montrer que cette proposition capte les données saillantes du français dans un cadre explicatif plus simple et plus proche de l'intuition que les traitements antérieurs. 


\title{
Définitude et quantification universelle
}

\author{
Francis Corblin \\ Université Paris-Sorbonne \\ \& Institut Jean Nicod (CNRS-ENS-EHESS)
}

Francis.Corblin@paris-sorbonne.fr

\section{Introduction}

Dans la plupart des théories du défini la notion de maximalité ou d'exhaustivité joue un rôle essentiel. Il est remarquable que cela ne distingue pas des théories d'inspiration très différentes. Par exemple, la théorie pragmatique de J.A. Hawkins (1978), se révèle très proche sur ce point de l'approche formelle de Link (1984) ; dans les deux théories la totalité ou la maximalité est au cour des interprétations définies. ${ }^{1}$ Et s'il est vrai que l'approche classique de B. Russell ne traite que du singulier en termes d'unicité, il est assez facile de voir que l'unicité est en fait une manifestation de la totalité dans des domaines d'interprétation particuliers (réduits à un élément).

La première question qui se pose à ce propos est d'ordre explicatif. Il y a après tout, d'autres formes linguistiques vouées à l'expression de la quantification universelle ${ }^{2}$, dans une langue comme le français , chaque, tout, tous les. Et il y a indiscutablement d'autres points de vue pour caractériser la définitude : on la rapproche souvent de l'anaphore, de la deixis, et on l'oppose à l'indéfinitude. Dans cette perspective, c'est le caractère connu, identifiable de la référence, qui est mis au premier plan, et non ce qui relève d'une quantification universelle. Cette première question se résume donc de la manière suivante : comment l'exhaustivité est-elle reliée à la théorie du défini? Une variante simplifiée de la question serait : doit-on considérer le défini, fondamentalement comme une forme linguistique particulière de la quantification universelle, ou la relation entre les deux notions est-elle une conséquence accidentelle d'une conception de la définitude fondée sur tout autre chose?

La seconde question qui se pose est d'ordre empirique. A peine a-t-on posé ou concédé que la définitude était, au moins en extension, équivalente à la quantification universelle, que les contre-exemples se présentent. Les définis pluriels s'emploient souvent alors que l'exhaustivité n'est pas visée.

(1) Les étudiants ont-ils accepté? Oui, mais pas tous.

L'objectif de cet article est de montrer que la seconde question peut être résolue de manière correcte pourvu que l'on traite la première. On soutient que la théorie du défini est indépendante, en principe de la notion de quantification universelle et d'exhaustivité.

Le défini est à analyser comme un terme, une expression linguistique référant à un individu singulier ou pluriel. L'exhaustivité va entrer en ligne de compte à deux titres :

1. Comme désignateur, terme référentiel, le défini atteint sa référence d'une manière qui implique l'exhaustivité, au moins relative à son domaine d'interprétation. Cela fournit une première source générant une certaine forme d'exhaustivité, mais ouvrant la voie au recouvrement partiel du domaine d'interprétation. Cette source est fondée sur la référence.

2. En tant qu'il désigne un individu (singulier ou pluriel) dont une propriété est prédiquée, le défini est concerné par une problématique plus générale. Lorsque l'on déclare qu'un individu vérifie une propriété, 
qu'en est-il des parties de cet individu? En admettant l'existence d'une ambiguïté entre des interprétations distributives et des interprétations collectives, on établira que chacune de ces deux interprétations offre des modalités différentes qui conduisent à s'affranchir de l'exhaustivité. Ces sources sont fondées sur la prédication.

\section{Les définis comme désignateurs}

Les principaux éléments de la théorie du défini introduits dans Corblin (1987) sont les suivants.

Si on interprète un GN comme défini, cela implique :

- qu'on l'interprète comme désignateur : le GN s'interprète comme un terme désignant un objet, catégorie dont le nom propre est le prototype, et à laquelle appartient également le démonstratif. Cette partie de la signification se glose comme : "Je désigne un objet du monde".

- qu'on l'interprète comme désignateur d'un type particulier (différent d'un nom propre et d'un démonstratif). Le mode de fonctionnement de ce désignateur peut être ainsi été résumé: «la description qui suit (contenu du GN) est le seul critère à utiliser pour extraire ma référence du domaine d'interprétation pertinent ».

De cette théorie, et de peu d'autres à ma connaissance, découle que l'exhaustivité, relativisée à un domaine d'interprétation, est une conséquence du mode de fonctionnement du désignateur. En gros, s'il est admis que le signalement "étudiant" est le seul critère à utiliser pour savoir de quoi je parle en utilisant "les étudiants", il suit que je vise tout ce qui est étudiant dans le domaine d'interprétation considéré. A cet égard, l'exhaustivité est obligatoire, et la seule manière d'y échapper relève de la délimitation du domaine d'interprétation, laquelle est déterminée pragmatiquement. Les étudiants désigne nécessairement tous les étudiants du domaine d'interprétation que le contexte de discours conduit à sélectionner. Imaginons le discours suivant :

(2) A cause du décret, les étudiants ${ }^{a}$ sont mécontents. D'ailleurs, dans notre campus les étudiants ${ }^{b}$ ont organisé une AG. Après cette réunion, il y a eu une manifestation en ville. Après la manifestation, la police a interpellé trois étudiants. Entre les policiers et les étudiants ${ }^{c}$ la discussion a été vive.

Il est tentant d'utiliser pour y décrire le défini le concept de caractère emprunté à D. Kaplan. Les groupes nominaux définis peuvent être caractérisés comme une fonction appliquée à des contextes différents pour rendre la dénotation de chaque emploi. La fonction caractéristique du défini serait en substance : "ce qui tombe sous d (contenu descriptif) dans le domaine d'interprétation sélectionné par C (contexte)". Dans l'exemple : ce qui est "étudiant", respectivement dans $C^{a}, C^{b}$, et $C^{c}$. Une telle caractérisation suppose implicitement que le domaine d'interprétation est sélectionné de manière univoque pour chaque contexte. On peut voir là une implicature liée à l'usage de tout désignateur. Si un désignateur a pour fonction de sélectionner pour mon interlocuteur un objet du monde dont j'entends dire quelque chose, lorsque j'en utilise un, je tiens pour acquis que cette fonction peut être remplie avec un degré de précision conforme à mes intentions.

Il se peut que j'attache une grande importance à la délimitation du domaine (et par là du référent). Il se peut au contraire que je n'y accorde pas une grande importance. Par exemple, dans la phrase initiale, parlé-je des étudiants en général, des étudiants français, de mon université, de mon UFR? Il est fréquent que nous ne nous préoccupions pas de préciser pour notre interlocuteur la limite maximale du domaine d'interprétation que nous prenons en compte. De multiples indices peuvent délimiter cet ensemble sur des critères de pertinence et de cohérence. "Dans notre campus", par exemple, délimite le domaine d'interprétation de les étudiants ${ }^{b}$; Pour les étudiants ${ }^{c}$, c'est le présupposé de continuité topique qui nous incite à admettre que le domaine d'interprétation à préférer est celui qui se limite aux individus mentionnés dans la phrase immédiatement antérieure.

La laxité des domaines est favorisée dans notre exemple par le fait qu'il s'agisse d'un pluriel faible (sans marque de cardinalité). Cette laxité est considérablement réduite par un cardinal, puisqu'il limite le choix du domaine à ceux qui ont $n$ éléments exactement. 
L'exhaustivité est donc, dans cette théorie, constitutive de la catégorie "défini" en raison même de son mode de désignation. Il est important, pour bien s'en persuader, de comparer avec le fonctionnement des désignateurs démonstratifs. Ces étudiants ne suppose en aucune façon que les référents du GN soient les seuls étudiants d'un domaine. Au contraire, de manière typique, on se ramène à un domaine d'interprétation où il y a d'autres étudiants.

Pour ce qui relève de la référence, donc, l'exhaustivité est inhérente; la seule manière dont on peut s'en libérer tient à la laxité des domaines d'interprétation du défini. Il faut aussi comparer à ce qui se passe pour la quantification universelle. Considérons tout $N$ comme paradigme de la quantification universelle linguistique. Il est vrai que ce quantificateur opère sur un domaine de quantification qui est souvent restreint par le contexte. Voir par exemple (3) :

(3) Dans notre université, tout étudiant a une adresse mail. Et dans mon cours, tout étudiant accède à une plate-forme interactive.

Une première différence semble être que la quantification universelle suppose par défaut le domaine maximal, et demande des indications explicites pour être limitée à une sous-partie de ce domaine. Le défini, en revanche semblerait plutôt prendre comme domaine, par défaut, ce qui est pertinent en contexte. Une preuve décisive est apportée par son fonctionnement anaphorique. Il suffit qu'une phrase d'un discours mentionne $\mathrm{x}$ étudiants pour que les étudiants, dans la phrase suivante, s'interprète sur un domaine où ces $x$ étudiants sont les seuls étudiants membres du domaine d'interprétation pertinent pour interpréter un défini.

La clé ici, pour comprendre la spécificité du défini est donc bien de caractériser avec précision ce qui distingue la construction dynamique des domaines d'interprétation pour le défini, et la restriction des domaines de quantification en contexte. Il semble bien que ces processus soient différents, mais nous ne considérons pas ce point ici pour des raisons d'espace. ${ }^{3}$

Dans tout ce qui suit, on considérera qu'il est possible de neutraliser cette variable concernant la dénotation du GN défini. On supposera, autrement dit, qu'il n'y a pas de doute sur ce que désignent les GN définis considérés dans les exemples. Une occurrence de « les étudiants, par exemple sera rapportée à un groupe d'étudiants donné, identifiable avec précision. Dans la mesure où pour nous, seul son mode de sélection d'un référent distingue le défini, tout ce qui va être dit s'applique en principe aussi bien aux désignateurs démonstratifs et aux désignateurs possessifs.

L'idée que nous développons dans ce qui suit est que même si la référence est identifiée avec précision, la prédication, l'attribution d'une propriété à une dénotation n'implique pas l'assignation de cette propriété à chacune des parties de cette dénotation.

\section{Défini et prédication}

Un désignateur sature un argument de prédicat en désignant une entité. L'interprétation résultante est que l'entité désignée par le désignateur satisfait le prédicat. Ici intervient une problématique dans laquelle l'exhaustivité va jouer son rôle. Quand on asserte qu'une entité satisfait un prédicat, il est seulement possible, et non nécessaire, que chaque partie de cet individu vérifie le prédicat. Pour certains prédicats, il n'est même pas requis, comme nous le verrons, qu'une partie quelconque de l'entité vérifie le prédicat. Nous appellerons ce type de prédication "individuel". Il est illustré par une phrase comme (4) :

(4) Sa robe était rouge

Mais il est possible également que l'entité désignée serve de domaine pour une quantification explicite, comme en (5):

\section{(5) Sa robe était (entièrement, partiellement) rouge}

Dans ce cas, il y a bien référence à une entité (la robe), mais les expressions entièrement et partiellement, indiquent quelle est la proportion des parties de cette référence qui satisfait le prédicat, jouant donc le rôle 
d'un quantificateur généralisé traitant cette dénotation comme un restricteur de quantification. Appelons ce type de prédication "quantificationnel".

En fait, la relation entre les deux types de prédications n'est pas trivial, comme attesté pas des exemples comme :

(6) Sa robe était rouge. Oui, mais pas entièrement

On ne peut donc pas, en général, à partir d'une prédication individuelle, déterminer quelles inférences à la prédicabilité des parties peuvent être tirées. Il est très net en particulier, sur le sujet qui nous occupe, qu'une inférence à la totalité des parties n'est pas garanti en général.

\subsection{Prédication individuelle et inférence aux parties}

Les inférences autorisées dépendent crucialement des prédicats impliqués. Voir sur ce point Carlson (1977), et beaucoup d'autres après lui (Dowty 1987, Kamp et Rossdeucher 1992, Yoon 1996, Löbner 2000, Rotstein et Winter 2004). J'utiliserai ici la typologie introduite dans Corblin (2008), typologie élaborée sur la base de ces travaux antérieurs.

On propose de distinguer :

1. des prédicats holistes : aucune inférence aux parties de l'individu n'est garantie.

(7) Ces étudiants sont nombreux

(8) Cette table est grande

2. des prédicats universels-majoritaires : l'inférence à chaque partie, ou au moins à la majorité, ou à la partie al plus saillante, est garantie.

(9) Cette robe est rouge

3. des prédicats existentiels : l'inférence à une partie au moins est garantie.

(10) Cette table est sale

La notion de prédicat holiste introduite dans Corblin (2008) consiste à traiter ces prédicats comme prédiquant une propriété d'un individu considéré dans la totalité de ses parties. L'idée est que ces prédicats sont restreints à la prédication individuelle, et ne permettent aucune forme de quantification sur les parties d'un individu. La classe des prédicats holistes inclut les prédicats de pure cardinalité de Dowty (1987), comme être nombreux, être quatre, mais est d'extension beaucoup plus large. La non-combinabilité avec les quantificateurs est illustrée par (10-11) :

\section{(11) *Ces étudiants sont tous nombreux}

\section{(14) *Cette table est entièrement grande}

Dans cette analyse, comme dans beaucoup d'autres (Scha 1981, Link 1983, Landman 1989) le défini pluriel peut être analysé comme référence à un groupe, intuitivement comme référence à une entité que l'on se représente comme un individu, perçu comme composé de parties du même type.

La prédication individuelle prédiquant d'un individu pluriel, l'inférence aux parties sera fonction des prédicats particuliers, lesquels sont associés à des postulats de signification qui fondent les typologies mentionnées plus haut. Ces postulats de signification offrent la possibilité d'avoir une très grande distance vis-à-vis d'une interprétation proche d'une quantification universelle sur les parties de la pluralité (notamment ses membres).

Ainsi, pour beaucoup de prédicats, notamment les prédicats "existentiels" on est réellement aux antipodes de l'exhaustivité. Considérons le verbe toucher employé avec un sujet pluriel comme en (15) :

(15) Les étudiants ont touché ce mur ${ }^{4}$ 
Il peut parfaitement être vérifié si un seul étudiant a touché le mur ${ }^{1}$. Il s'agit d'une prédiction directe de cette approche : le prédicat est dit vérifié par un individu pluriel, un groupe. Le prédicat étant par ailleurs de type "existentiel", l'inférence est seulement qu'une partie au moins de ce groupe le vérifie. Le fait qu'il s'agisse d'une propriété lexicale de "toucher" est confirmé par le fait que cette propriété vaut également si le sujet est singulier ; ainsi, (15) est vrai si une partie de Pierre touche le mur :

\section{(16) Pierre touche le mur}

Une telle approche est par conséquent en mesure de rendre compte sans difficulté des cas où définitude et quantification universelle donnent lieu à des conditions d'emploi et à des conditions de vérité diamétralement opposées, comme en (17) :

\section{Le peintre : Les étudiants ont touché le mur.}

Un observateur: Oui, mais une seul d'entre eux l'a fait.

Le point est très exactement qu'une prédication individuelle ne préjuge pas, pour ce prédicat, que chacune de ses parties le vérifie, mais seulement qu'il existe une partie au moins qui le vérifie.

Le problème est que beaucoup des exemples considérés, y compris (15) sont perçus comme ambigus. Les analyses qui précèdent semblent bien s'appliquer aux interprétations dites"collectives" de ces définis, ce que nous analysons ici comme des prédications individuelles. Cependant, pour beaucoup de chercheurs, et même en l'absence de toute forme lexicale interprétable comme un quantificateur, ces exemples ont aussi une interprétation "distributive" laquelle semble épouser de beaucoup plus près les conditions d'emploi des formes linguistiques de la quantification universelle. En outre, les GN définis peuvent se combiner avec des expressions analysables, au moins à première vue comme des quantificateurs, tels que tous.

\subsection{Interprétations distributives}

Les chercheurs ne sont pas unanimes quant à la nécessité d'admettre une ambiguité collectif/distributif pour le pluriel, mais le point de vue dominant est qu'il est impossible de traiter les interprétations distributives comme un cas particulier d'inférence compatible avec l'interprétation du sujet comme "groupe" (voir par exemple Roberts (1987), et Landman (2000)). Il faut donc postuler une ambiguité.

Dans la plupart des théories, l'interprétation "distributive" est assimilée à la quantification universelle de la logique du premier ordre. Les théories se partagent quant à l'origine de cette quantification universelle.

Pour certains auteurs, le groupe nominal est ambigu : il admet une interprétation "groupe" et une interprétation distributive : cela revient, pour la théorie du défini, a considérer, que dans certains emplois, le défini est une forme de la quantification universelle du premier ordre. C'est le cas dans les théories de Scha (1981) et de Bennett (1974)). Pour d'autres, en particulier pour Link (1983), c'est le prédicat qui est ambigu : il peut s'interpréter ou comme une prédication individuelle, ou comme une quantification universelle. On postule donc que certains prédicats s'interprètent éventuellement dans le champ d'un quantificateur universel invisible «D $»$.L'argument le plus souvent retenu contre l'ambiguïté du GN repose sur la possibilité de coordonner des définis collectifs et distributifs comme dans (18) qui coordonne, un prédicat holiste collectif (être nombreux) et un prédicat strictement atomique distributif(être barbu) :

\section{(18) Les étudiants étaient nombreux et barbus}

L'argument est que la source d'une quantification universelle du premier ordre ne peut pas être le défini lui-même, sinon il propagerait cette quantification aux deux prédicats coordonnés, alors qu'un seul d'entre eux admet une telle quantification. Cependant, pour rendre compte de ces deux sortes de prédication (individuelle et quantification universelle), il est nécessaire de considérer que l'individu auquel réfère le défini peut être traité ou comme référence à un individu (un groupe pour la prédication collective), ou 
référence à un ensemble (jouant le rôle d'un domaine de quantification pour le quantificateur universel distributif supposé), cf. Corblin (2008). Il est alors difficile de dire le prédicat seul "responsable" de l'interprétation distributive. C'est plutôt la prédication, dans son ensemble qui est la source du processus. L'idée défendue dans Corblin (2008) est que si le GN défini s'interprète comme référence à un ensemble d'éléments et non à un groupe, la prédication ne peut s'effectuer que par l'intermédiaire d'une quantification implicite, ou explicite. Il est peut-être de bonne méthode, par conséquent, d'étudier d'abord les combinaisons des GN définis avec des quantificateurs explicites.

\subsection{La combinaison du défini et de tous. Prédication quantificationnelle.}

En dehors des prédicats holistes (voir supra), la combinaison du défini et de tous, préfixé ou flottant, est parfaitement licite.

\section{(19) Les étudiants ont tous touché le mur}

Tous apparaît en outre ici comme élément d'une classe, celle des expressions quantifiées comme en témoigne (20) :

(20) Les étudiants ont (en majorité, par dizaines) touché le mur

L'article pionnier de Dowty (1987) soulève à propos de cette combinaison deux questions essentielles :

A. Pourquoi all (tous) ne se combine-t-il pas avec certains prédicats ? Voir (21).

B. Pourquoi peut-il se combiner avec des collectifs ? Voir (22).

(21) *Les étudiants sont tous nombreux

(22) Les étudiants se rassemblent tous dans la cour

La réponse de Dowty est fondée sur la prémisse implicite suivante : tous est un quantificateur universel du premier ordre, il assigne une propriété à chacun des individus atomiques dénotés par le sujet.

La prédication échoue en (21) parce que ces prédicats («pure cardinality predicates ») peuvent être vérifiés par une collection d'individus sans que l'on puisse en inférer la moindre propriété assignable aux éléments. Il ne se trouve donc aucune inférence («sub-entailment») à distribuer sur chacun des individus de l'ensemble. En revanche, un prédicat comme se rassembler ne peut pas être vérifié par une collection sans que certaines propriétés soient vérifiées par ses éléments (par exemple se déplacer en convergeant,...$)$; il est donc possible d'interpréter le quantificateur comme distribuant cette propriété sur chacun des éléments de l'ensemble.

La réponse de Dowty ajoute à la prémisse initiale la nécessité de recourir à la notion de sub-entailment, laquelle semble problématique (voir par exemple Brisson (2003))

Corblin (2008) suggère une autre réponse à ces questions qui rejette la prémisse implicite et ne recourt pas à la notion de «sub-entailment ». Tous est analysé comme un quantificateur généralisé opérant sur une partition de la dénotation, et non comme un quantificateur du premier ordre. Pour un prédicat comme se rassembler, qui ne s'applique qu'à une pluralité, tous indique que chaque élément d'une partition de la dénotation de «les étudiants 》 satisfait le prédicat se rassembler. Et est seule concernée une partition de cette pluralité en pluralités, puisque le prédicat ne s'applique qu'à de telles entités. Si tous ne peut pas se combiner avec les prédicats holistes, c'est que ceux-ci s'appliquent à un individu « considéré dans toutes ses parties » et n'impliquent aucune inférence aux parties (cf.supra). On montre dans ce travail que ces prédicats holistes ne sont pas liés à l'expression de la cardinalité, comme le suggère Dowty, mais que la notion est d'extension très large, et non limitée au pluriel, comme en témoignent (23) et (24) :

(23) Pierre pèse (*entièrement) lourd

(24) La cuisine est (*entièrement) grande 
Un point important de cette approche est que tous est bien l'expression lexicale d'une quantification, mais qu'il ne s'agit pas d'un quantificateur du premier ordre, donc qu'il ne quantifie pas nécessairement sur des individus.

Il importe de considérer la manière dont tous va se combiner avec les différents types de prédicats habituellement distingués soit :

Prédicats atomiques : être enceinte, être barbu.

Prédicats collectifs : se rassembler, se disperser

Prédicats mixtes : acheter, transporter

En gros, les prédicats atomiques ne peuvent pas être prédiqués d'un groupe (*Le comité est enceinte), les prédicats collectifs ne peuvent pas être prédiqués d'un atome (*Marie se rassemble); les prédicats mixtes n'ont pas de contrainte de combinaison.

Nous admettons que la signification de tous est que la totalité des parties de l'individu satisfait le prédicat. Les prédicats atomiques vont induire nécessairement que la partition considérée pour une dénotation plurielle est une partition en individus (puisque ces prédicats ne s'appliquent qu'à des individus), ce qui induit nécessairement une interprétation "distributive", comme illustré par (25) :

\section{(25) Les étudiants sont tous barbus}

On notera le contraste avec (26) :

\section{(26) ? Les étudiants avaient (tous) une barbe chacun}

Chacun semble explicitement exiger que le prédicat soit appliqué à tous les atomes d'une pluralité pris un à un. Or pour un prédicat comme être barbu, par sa définition de prédicat atomique, il ne peut en être autrement, d'où sans doute le caractère comique de l'exemple, par «redondance ». Si tous, en revanche, est parfaitement naturel en combinaison avec de tels prédicats, c'est, comme on le suppose ici, que rien dans sa signification n'impose une quantification sur les atomes ; tous indique seulement que la totalité des parties de l'ensemble dénoté satisfait le prédicat. Il est typique que tous peut s'employer sans difficulté dans des phrases comme les lions ont tous une queue, alors que des phrases comme les lions ont chacun une queue est comique. Le point semble être que pour un prédicat "strictement atomique", comme avoir une queue, le recours à chacun revient à asserter que le prédicat est vérifié par chacun des atomes. En revanche, tous ne possède aucun élément de signification assertant que l'on a affaire à une propriété atomique : il asserte seulement que toutes les parties de l'ensemble vérifient la propriété.

Les prédicats collectifs, quant à eux, sélectionnent des partitions en individus pluriels et tous asserte que chacun de ces individus pluriels vérifie le prédicat. Une formulation forte de cette analyse serait que dans toute partition de l'individu pluriel en parties plurielles, chacune des parties vérifie le prédicat. L'idée serait que dans un énoncé comme les étudiants se rassemblent tous dans la cour, pour toute partition des étudiants en groupes, chacun de ces groupes vérifie le prédicat se rassembler. Une version plus faible serait qu'il existe une partition du groupe en sous-groupes telle que chacun des sous groupes vérifie le prédicat. C'est cette version que nous adoptons ici.

Pour les prédicats mixtes, tous indique que chacune des parties d'une partition de l'individu pluriel satisfait le prédicat. C'est ainsi que s'interprète, par exemple, une phrase comme :

(27) Mes amis ont tous acheté une maison

Contrairement à ce qu'on admet parfois, cette phrase ne signifie pas que chacun de mes amis a acheté sa propre maison. Elle sera jugée vraie, par exemple, si j'ai 5 amis, Albert, Nicole, Louis, Emma, et Ludo, que Albert et Nicole aient acheté en commun une maison et que Louis, Emma et Ludo aient acheté la leur. 
Le contraste entre tous et chacun, dans ce contexte où les deux peuvent être employés, est assez net. Dans Mes amis ont chacun acheté une maison, il est requis, d'une part que les amis soient considérés individuellement, et d'autre part que l'indéfini soit interprété dans la portée du quantificateur universel. Aucune de ces deux propriétés ne vaut pour tous.

Un problème surgit avec des exemples tels que (28)

\section{(28) Mes amis m'ont tous offert ce magnifique tableau}

(28) admet en effet une interprétation classiquement appelée " collective ». Il est possible d'utiliser cette phrase alors qu'un seul cadeau a été fait, offert collectivement par mes amis. Cette probabilité augmente d'ailleurs si tous n'est pas flottant mais en position de prédéterminant :

\section{(29) Tous mes amis m'ont offert ce magnifique tableau}

Apparemment, cette interprétation contredit l'idée que tous est un quantificateur généralisé impliquant que la propriété est assignée aux parties du groupe, puisque l'interprétation semble l'assigner au groupe entier, et à lui seulement. Il s'agit donc d'un vrai problème pour l'analyse proposée, puisque tous y semble compatible avec une interprétation collective du terme auquel il s'applique, alors que nous proposons de dire qu'il asserte que la propriété est vérifiée par chaque partie d'une partition.

Il y a deux réponses qui viennent à l'esprit immédiatement. On peut mettre en doute, empiriquement, le fait que (28) soit parfaitement acceptable. Il est vrai que l'on trouve des locuteurs qui disent ne pas aimer cette phrase. On peut aussi tenter de jouer sur la notion de partition en admettant des partitions nonstrictes : le groupe total lui-même étant une partie de lui-même. ${ }^{5}$

Mais cela ne rend pas compte de ce que beaucoup de locuteurs acceptent (28-29), et avec une sémantique spécifique : on utilise (28-29) pour indiquer que chacun des amis a participé, a joué un rôle actif pour faire ce cadeau, alors que la version sans tous indique seulement que le groupe, comme un tout, a fait ce cadeau.

Supposons que nous décidions de traiter (28-29) comme une véritable interprétation quantifiée, soit : il existe une partition de l'ensemble de mes amis telle que chacune de ses parties m'a acheté ce magnifique tableau. Simplifions même en considérant une partition en individus, ce qui peut se représenter comme suit :

$$
\begin{aligned}
& \text { a m'a acheté ce magnifique tableau } \\
& \text { b m'a acheté ce magnifique tableau } \\
& \text { c m'a acheté ce magnifique tableau }
\end{aligned}
$$

Le problème est, en substance, que si nous admettons a m'a acheté ce magnifique tableau, alors nous considérons que $b$ n'a pas pu le faire, ni $c$, etc... Quelle est la source du problème? Nous admettons en fait que pour certaines prédications $\mathrm{P}$, asserter $\mathrm{P}(\mathrm{a})$ a pour implicature que aucun autre individu ne vérifie $\mathrm{P}$. On en trouve trace dans certaines inférences naturelles telles que : si $x$ a tué la victime, alors y ne l'a pas tuée, etc.

Cela pourrait nous donner la clé du statut de (28-29). Il existe bien une interprétation quantifiée, signifiant que le groupe contient ceux qui ont pris part au don. Cette interprétation est refusée par certains locuteurs pour la même raison que ces locuteurs refuseraient Pierre a acheté des fleurs à sa mère, dans le cas où Pierre et sa sœur se partagent l'addition de la fleuriste. Le point est que l'interprétation visée suppose que $l^{\prime}$ 'on utilise l'expression sans son implicature habituelle selon laquelle $\mathrm{P}(\mathrm{a})$ implique qu'on n'a $\mathrm{P}(\mathrm{x})$ pour aucun $\mathrm{x}$ diférent de $\mathrm{a}$.

Le problème est beaucoup plus général que celui qui nous occupe ici. Considérons par exemple :

(30 Pierre finance (soutient) l'UMP 


\section{(31) Cet homme dirige l'UMP}

Il n'est aucunement impliqué que Pierre finance à lui seul (que personne d'autre ne le fait) l'UMP. En revanche, il y a une implicature forte selon laquelle personne d'autre que cet homme ne dirige l'UMP. un contraste de ce type confirme que l'implicature est une propriété lexicale, qui distingue les prédications. Il est facile de voir, par exemple, que lire un livre est dépourvu de cette implicature, alors qu'écrire un livre en est pourvu.

Offrir $x$ est sans aucun doute une prédication qui déclenche l'implicature (et personne d'autre n'a offert x) ce qui permet de rendre compte à la fois de l'interprétation attestée en (28-9), et également du fait qu'elle apparaisse comme marginale à certains locuteurs.

Dans cette optique, (28-29) est une véritable interprétation quantificationnelle. (28-29) affirme que la totalité des parties de l'ensemble «mes amis » a fait ce don (au sens de "pris part à ce don)". Cette interprétation est différente de l'interprétation « individuelle » postulée $§ 2$ supra et illustrée par (32) :

(32) Mes amis m'ont offert ce magnifique tableau

Dans cette interprétation, c'est l'individu-groupe mes amis qui est le donateur, et rien n'est dit de la participation de tous les éléments de ce groupe à l'action.

Il est à noter que l'analyse que nous proposons ici de l'interprétation du contraste (28-32) est différente de ce que proposent Link (1983, pp 310-311) et Dowty (1987).

Link, pour traiter d'exemples correspondants à (28) utilise un prédicat ad hoc "to take part in" donné comme une primitive nécessaire à l'interprétation de all. Ce prédicat donne, grossièrement le résultat attendu : (28) signifie que chaque membre du groupe a pris une part au don, alors que (32) indique seulement qu'un don a été fait par le groupe pris comme un tout. Le problème majeur est justement le recours à une primitive ad hoc pour l'interprétation de all. Dans la présente analyse, tous impose que le don soit imputé à chaque partie du groupe, ce qui n'est compatible qu'avec une interprétation 'prendre part à". Cette interprétation "prendre part à" n'est pas ad hoc, mais au contraire nécessaire par ailleurs, puisqu'il faut y avoir recours pour expliquer des phénomènes aussi courants que : Le père paie pour les études de sa fille, mais la mère aussi.

Dowty (1992) traite d'exemples similaires avec all en termes de sous-implications (sub-entailment). (32) indique qu'un don collectif a été fait, ce qui implique seulement qu'un sous groupe de mes amis a joué un rôle causal dans le don, mais par exemple, Pierre, un de mes amis, a peut-être, par avarice ou étourderie oublié de payer sa part : (32) reste néanmoins vraie. All, pour Dowty doit distribuer des sous-implications à chaque individu du groupe : il propose d'admettre que tous distribue la sous-implication distributive d'être membre du sous-groupe qui agit causalement dans l'accomplissement du don. Si Pierre n'a rien payé ni rien fait pour ce don, (28) sera fausse. A nouveau nous avons affaire à une analyse qui produit le même résultat que celle que nous défendons. La différence est là encore que nous notre proposition fait l'économie du caractère distributif de tous et de la notion de sub-entailement, et qu'en outre elle rend compte du caractère marqué de ce type d'exemple.

\subsection{Prédication quantificationnelle sans quantificateur}

Comme indiqué $\S 3.2$, il y a des interprétations du seul défini (sans quantificateur) qui sont clairement interprétées comme quantifications (universelles ou quasi-universelles), notamment les interprétations « distributives ».

La plupart des auteurs placent l'origine de ces interprétations distributives dans l'interprétation du prédicat, non dans l'interprétation du défini lui-même, et les engendrent au moyen d'un quantificateur universel du premier ordre « implicite ».

Cette approche soulève un certain nombre de difficultés:

a) Multiplication des quantificateurs. 
Supposons que l'on postule un quantificateur universel distributif pour justifier que (33) soit distributif :

\section{(33) Les étudiants m'ont offert un cadeau}

Si l'on adopte la thèse qu'en (33), le défini lui même, comme dans toutes ses interprétations (distributives ou non), s'interprète comme quantification universelle, cela conduit à avoir en (33) deux expressions de la quantification universelle dont aucune n'est lexicalement réalisée. C'est en fait le contenu du traitement de Link (1983). Si on ajoute tous, ce qui est parfaitement licite, le nombre de quantifications universelles serait de trois (dont deux implicites).

b) Problèmes de justification empirique.

Le postulat d'un quantificateur universel distributif «invisible » est ad hoc, et tel quel non relié à une autre partie de l'analyse sémantique de la langue.

Pour tenter d'éviter ces problèmes, l'analyse présentée ici ne fait pas de la quantification universelle une composante de la signification du défini. Le défini est analysé comme référence à un individu, singulier ou pluriel.

D'autre part, nous n'analysons pas tous comme un quantificateur du premier ordre, mais comme un quantificateur généralisé universel qui quantifie sur les parties d'un individu (atomiques ou non).

Nous avons précisé la différence entre cette analyse et celle de Dowty (1987). Il faut souligner que cette analyse est également différente de celle de Brisson (2003) laquelle est inspirée de Swartschild (1994). Pour l'essentiel, Brisson analyse le défini comme un quantificateur universel «faible », c'est à dire autorisé à ne pas prendre en compte la totalité du domaine de discours. Le problème pour ce type d'analyse réside dans la signification qu'elle est amenée à proposer pour l'élément tous qui se combine avec le défini. Si tous est analysé comme quantificateur universel, il est redondant, et donne éventuellement lieu à des phrases comportant trois universels, dont deux invisibles (celui du défini, et celui du distributif). Aussi n'est-il pas analysé comme un véritable quantificateur par Brisson, mais plutôt comme un "réducteur de marge", dans l'esprit de Lasersohn (1999). L'idée est que la communication se satisfait souvent très bien d'approximations, et dispose d'outils linguistiques pour indiquer que ces zones d'approximation sont réduites. Par exemple, si on me dit Je viendrai à cinq heures, je comprends que l'heure d'arrivée sera située dans un temps qui englobera cinq heures, et dont l'extension n'est pas donnée exactement. Si en revanche on me dit Je viendrai à cinq heures exactement, "exactement" réduit cette zone d'approximation à néant; exactement est analysé comme un réducteur de marge. Les analyses du défini fondées sur cette théorie en font un quantificateur universel, qui s'accommode d'une zone d'approximation. En outre, les théories considérées situent la zone d'approximation non dans la signification elle-même, mais dans l'extension du domaine de quantification sur lequel quantifie l'universel. Le problème principal que soulèvent ces théories, de mon point de vue, est qu'elles doivent traiter tous (all) comme un simple réducteur de marge, en gros de même signification que "exactement". Il s'agit d'une solution qui ne me paraît pas très convaincante intuitivement. Il serait préférable de travailler dans une théorie où tous signifie ce qu'il paraît signifier, c'est à dire la quantification universelle.

Il reste à examiner comment la présente approche accommode les phrases sans quantificateur en évitant les problèmes mentionnés.

Commençons par la justification empirique. Dans Corblin (2002) un rapprochement a été opéré avec l'analyse que fait D. Lewis des conditionnelles dans son article de 1975. Lewis y affirme que les conditionnelles sont des phrases quantificationnelles sans adverbe de quantification lexicalement exprimé. Ainsi, pour Lewis, les deux phrases suivantes sont des variantes stylistiques : 


\section{(35) S'il pleut, je prends toujours mon parapluie}

Grossièrement, l'idée est que si une phrase se présente sans ambiguïté comme une phrase quantifiée identifiant un restricteur et un scope nucléaire, en l'absence d'un quantificateur explicite, elle s'interprète comme une quantification universelle. Lewis (1975) traite donc comme "variation stylistique" la variation: "(Always) When it trains, it pours" . Il insiste également sur le fait que "if" ne doit pas être traité comme un connecteur phrastique (logique) : "[if] has no meaning apart from the adverb it restricts". Il suit de cela que dans une phrase sans adverbe de quantification explicite comme "toujours", "parfois",..., mais comportant une structure si $p q$, l'antécédent $p$ s'interprète comme un restricteur de quantification, $q$ comme scope nucléaire. Dans ce cas, dit Lewis qui ne s'en explique pas davantage, "the variables are bound by the ommitted always".

Lewis ne commente pas en détail ce point particulier. L'idée que j'ai défendue dans les travaux cités est que si un quantificateur explicite n'est pas présent, dans une structure interprétable comme quantification, on interprète la quantification comme universelle ou quasi universelle, des facteurs d'ordre pragmatique opérant un choix entre ces deux options. ${ }^{6}$

Il est facile de montrer que le rapport à l'universalité stricte est exactement le même pour les conditionnelles et les définis qui nous occupent. Dans un type de discours qui considère en général des propriétés strictement universelles (mathématiques, droit, par exemple), un quantificateur non exprimé donnera lieu à une quantification universelle stricte : si un nombre est pair, il n'est pas premier; les nombres pairs ne sont pas premiers. Dans un type de discours où les généralisation absolues sont rares (morale, vie quotidienne) on interprétera plus volontiers l'absence de quantification comme "en règle générale" : Si un enfant réussit, il aime travailler: les enfants qui réussissent aiment travailler.

Je propose donc de considérer que dans la prédication, les définis, en l'absence de toute forme de quantification lexicalement réalisée, peuvent offrir matière à une prédication quantificationnelle: on interprète alors la phrase comme quantification universelle ou quasi-universelle. La différence entre la version comportant tous est que dans ce cas, un quantificateur généralisé universel opère la quantification. Ce quantificateur généralisé «implicite » est la seule origine des interprétations distributives, et on n'a donc pas, dans cette perspective de problème de multiplication des quantificateurs ni de problème pour justifier un opérateur distributif invisible ad hoc. L'interprétation distributive stricte (chaque individu vérifie le prédicat) est simplement une des possibilités d'interprétation d'une quantification universelle sur les parties de l'ensemble, et compte donc au nombre des interprétations possibles de tous. On ne discutera pas ici du contraste tous/chacun qui a été largement documenté dans la littérature. ${ }^{7}$

Il est utile de préciser clairement ce qui distingue cette approche de la solution de Brisson. Pour elle, le défini est un quantificateur universel faible, autorisé à ne pas prendre en compte tous les éléments dans le domaine ; tous est un opérateur qui prive le défini de cette liberté. Pour la présente proposition, le défini dénote un individu et ne contient aucune signification quantificationnelle. Il y a donc des prédications définies strictement non-quantificationnelles (ce que nous avons appelé des prédications individuelles). Le défini, s'il est traité comme un ensemble de parties offre matière à une prédication quantificationnelle, laquelle, en l'absence de tout quantificateur, est réalisée par un quantificateur généralisé interprété comme universel ou quasi-universel; tous est un quantificateur généralisé qui quantifie sur les parties d'un individu pluriel.

Pour nous, les avantages de cette solution sont les suivants. L'analyse proposée fait usage des mêmes principes que ce qui est nécessaire selon Lewis pour les conditionnelles, notamment du principe selon lequel une structure de quantification sans quantificateur réalisé s'interprète au moyen d'une quantification «universelle ou presque universelle» selon la nature du discours considéré. Nous maintenons d'autre part à tous l'interprétation que lui confère l'intuition: il s'agit d'une forme de la quantification universelle. Enfin, cette hypothèse suffit à générer sans autre stipulation les interprétations distributives : le quantificateur explicite, ou implicite est un quantificateur universel sur les parties de l'individu qui admet comme cas possible une quantification sur les atomes constitutifs, cette dernière interprétation étant exprimée par chacun. 


\section{Conclusion. Résumé et discussion}

Cette communication soutient que la définitude est par nature indépendante de la quantification universelle. La définitude implique seulement l'interprétation comme désignateur pourvu d'un mode d'identification de la référence spécifique.

Ce mode d'identification détermine un premier lien à l'universalité : le défini le $\mathrm{x}$ désigne nécessairement tous les $\mathrm{x}$ de son domaine d'interprétation; ce sont les limites non-contraintes de ce domaine qui l'éloignent de l'universel strict.

En tant que désignateur, et au même titre que le nom propre, le défini entre dans la prédication, ce qui détermine d'autres associations à l'universalité.

Le défini réfère à un individu et il existe un mode de prédication « individuelle » qui assigne le prédicat à l'individu dénoté. Les inférences possibles relatives à la satisfaction du prédicat par les parties de sont déterminées, prédicat par prédicat, selon la classe à laquelle ce prédicat appartient (existentiel, majoritaire, holiste).

Le défini réfère à un individu qui peut être vu comme un ensemble de parties et fait l'objet d'une prédication quantifiée au moyen d'une expression lexicale telle que tous. Seuls les prédicats holistes qui s'appliquent à un individu considéré dans toutes ses parties n'admettent pas la prédication quantificationnelle.

Le défini, comme les conditionnelles et comme l'indéfini générique, admet des interprétations quantificationnelles sans quantificateur lexical réalisé. Comme pour les conditionnelles $(s i P Q)$ et l'indéfini générique, le quantificateur restitué s'interprète comme « universel ou quasi-universel ».

\section{Références bibliographiques}

Anscombre, J.C. (2006) «Tout, n'importe quel, chaque: quelques remarques», dans Indéfini et prédication, Corblin, F., Ferrando, S., Kupferman, L. (eds), PUPS, pp. 431-448.

Bennett, M. (1974) Some extensions of a Montague Fragment of English, PhD. Dissertation, UCLA.

Brisson, C. (2003) «Plural, «all», and the nonuniformity of collective predication», in Linguistics and Philosophy, pp.129-184.

Comorovski, I. (2003) « On the emergence of Purely Collective Predication », in P. Dekker \& R. van Rooy, eds, Proceedings of the 14th Amsterdam Colloquium, ILLC, University of Amsterdam.

Comorovski, I. \& Nicaise, L. « divers », in Corblin F. \& de Swart, H. (eds), Handbook of French Semantics, CSLI, Stanford, pp. 119-131.

Corblin (1987) Indéfini, défini et démonstratif, Droz, Genève.

Corblin, F. (2002) Représentation du discours et sémantique formelle. Introduction et application au français, PUF.

Corblin, F. (2008) «Des prédicats non quantifiables : les prédicats holistes», Langages 169, pp. 34-57.

Dowty, D. (1987) «Collective predicates, distributive predicates and all»,F. Marshall,ed. Proceedings of the 3rd ESCOL, Ohio state university.

Galmiche, M. 1977) «Quantificateurs, référence et théorie transformationnelle», dans Langages, 11,48, pp 3-49. 
Kamp, H. Roßdeutsher, A. (1992) "Remarks on Lexical Structure, DRS-Construction and Lexically Driven Inference», Research papers, Bericht 21, Stuttgart.

Kleiber, G. \& Martin, R. . (1977). «La quantification universelle en français. » Semantikos 2, pp. 19-36.

Landman, F. (1989) «Groups I», «Groups II», Linguistics and Philosophy, 12.5;12.6.

Landman, F. (1996) «Plurality», in The Handbook of Contemporary Semantic Theory, Lappin, S. (ed.) Blackwell, pp.423-425.

Landman, F. (2000) Events and Plurality, Kluwer.

Lappin, S., ed. (1996) The Handbook of Contemporary Semantic Theory, Oxford, Blackwell.

Lasersohn, P. (1995). Plurality, conjunction and events Dordrecht: Kluwer.

Lasersohn, P. (1999), «Pragmatic hallos », in Language, Vol. 75-3, pp 522-551.

Le Querler, N. (2006) «Les déterminants indéfinis tout, quelque, chaque,certains et la prédication de propriété» dans Indéfini et prédication, Corblin, F., Ferrando, S., Kupferman, L. (eds), PUPS, pp. 349-369.

Lewis, D. (1975) «Adverbs of quantification», in Keenan , E.L. (1975) Formal Semantics of Natural Language, Cambridge University Press.

Link, G. (1983) «The Logical Analysis of Plurals and Mass Terms. A Lattice- Theoretic Approach», reproduit dans Portner, P., Partee, B., eds (2002) Formal Semantics. The essential readings, Blackwell, Oxford.

Löbner, S. (2000) «Polarity in Natural Language :Predication, Quantification and Negation in Particular and Characterizing Sentences », Linguistics and Philosophy 23 : 213-308.

Martin, F. (2006) Prédicats statifs, causatifs et résultatifs en discours, thèse ULB.

Roberts, C. (1987) Modal Subordination, Anaphora and Distributivity, Ph.D. Thesis Massachussetts Un.

Rotstein, C. \& Winter, Y. (2004) "Total Adjectives vs. Partial Adjectives : Scale Structure and Higher-Order Modifiers, Natural Language Semantics $12: 259-288$.

Scha, R. (1981) «Distributive, collective and cumulative quantification », in J. Groenendijk, T. Jansen, and M. Stockhof, eds. Formal Methods in the Study of language, Mathematical Center tracts, Amsterdam.

Schwarzschild, R. (1996) Pluralities, Kluwer, Dordrecht.

Yoon, Y. (1996) "Total and Partial Predicates and the Weak and Strong Interpretation ", in Natural Language Semantics 4, 3, pp.217-236.

\footnotetext{
${ }^{1}$ Selon Hawkins «...definite descriptions refer to the totality of the objects, or to the whole mass, in the relevant shared set... »(1978, p. 159) : Link (1983) analyse le défini pluriel comme l'élément maximal du treillis des sommes $(« \sigma »)$.

${ }^{2}$ Voir par exemple Kleiber \& Martin (1977) et de nombreux travaux ultérieurs.

${ }^{3}$ Il y a sans aucun doute ici à discuter de la différence entre tout $N$, expression formelle de l'universalité, et tous les $N s$, version beaucoup plus naturelle (au sens de langue "naturelle") de l'universalité. Il est difficile de le faire ici avant de caractériser plus précisément la configuration « tous les Ns, les Ns tous », qui est précisément l'objet de cet article.

${ }^{4}$ Un relecteur anonyme de l'article note que (15) est pour lui peu naturel si un seul étudiant a touché le mur. Il mentionne cependant que feraient exception des cas où un groupe est traité comme une unité, par exemple, au rugby une phrase comme 'les joueurs ont touché la ligne". Nous avons noté ailleurs (corblin 2008) à quel point de telles phrases, qui imputent à une pluralité ce qui est le fait d'un seul prêtent à discussion parmi les locuteurs, dans la vie réelle, et pas seulement parmi les linguistes. Notre exemple favori est celui d'une inscription dans le hall d'un lycée : bien qu'il sache qu'une seule main a opéré, le proviseur dira sûrement "Les étudiants ont taggué le hall". Si on lui objecte qu'une seule personne a fait le coup, il l'admettra, mais il n'est pas sûr qu'il considérera sa phrase comme fausse. Peut-être considérera-t-il seulement que la version au singulier "Un étudiant a taggué le hall" est également vraie et que le choix de l'une ou l'autre est une matière de pragmatique.
} 
ISBN 978-2-7598-0358-3, Paris, 2008, Institut de Linguistique Française

Sémantique

DOI $10.1051 / \mathrm{cmlf0} 0128$

\footnotetext{
${ }^{5}$ Un relecteur anonyme de l'article envisage lui-même cette possibilité, qui ne me semble cependant pas une réponse suffisante pour les raisons considérées infra.

${ }^{6}$ Le cas de l'indéfini générique est une application directe de ce principe. Dans une phrase comme Un chien aime son maître, il n'y a aucun item lexical qui signifie que cette phrase est universelle. Il peut y en avoir un (Un chien aime toujours son maître). Lorsqu'il n'y en a pas, l'interprétation est universelle, ou quasi-universelle en vertu du principe évoqué ici. C'est la raison pour laquelle on a à juste titre noté que l'indéfini admettait des exceptions, alors qu'une expression lexicale de la totalité comme Tout chien aime son maître les admet plus difficilement.

${ }^{7}$ Pour une approche récente voir notamment Anscombre (2006), Le Querler (2006).
} 